Cahiers
de la Recherche
sur les Droits

Cahiers de la recherche sur les droits

fondamentaux

Fondamentaux

12 | 2014

Droit et psychiatrie

\title{
Des lois sous influences
}

Jean-Manuel Larralde

\section{OpenEdition}

Journals

Édition électronique

URL : http://journals.openedition.org/crdf/1868

DOI : $10.4000 /$ crdf. 1868

ISSN : 2264-1246

Éditeur

Presses universitaires de Caen

Édition imprimée

Date de publication : 1 décembre 2014

Pagination : 19-27

ISBN : 978-2-84133-507-7

ISSN : $1634-8842$

Référence électronique

Jean-Manuel Larralde, "Des lois sous influences », Cahiers de la recherche sur les droits fondamentaux [En ligne], 12 | 2014, mis en ligne le 01 octobre 2015, consulté le 11 février 2020. URL : http:// journals.openedition.org/crdf/1868; DOI : 10.4000/crdf.1868 


\title{
Des lois sous influences
}

\author{
Jean-Manuel LARRALDE \\ Professeur de droit public à l'université de Caen Basse-Normandie \\ Directeur adjoint du Centre de recherche sur les droits fondamentaux et les évolutions du droit (CRDFED, EA 2132)
}

\section{Limiter les hospitalisations sans consentement à des cas "légitimes " mis en œuvre par des techniques "proportionnées"}

II. Des hospitalisations sans consentement effectivement contrôlées: la place essentielle du juge

III. La question de la dignité de la personne soumise à des soins sans consentement

On le sait depuis Montesquieu, les lois ont un « esprit» ${ }^{1}$. Elles sont effectivement le produit de facteurs multiples, sociaux, politiques, économiques ou juridiques, qu'il est parfois difficile, mais parfois plus aisé d'identifier de manière précise. À cet égard, la loi du 5 juillet $2011^{2}$, comme la loi du 27 septembre $2013^{3}$, appartiennent à la deuxième catégorie: elles ont en effet été l'œuvre d'un législateur travaillant sous la pression constante du Conseil constitutionnel, puisque ce ne sont pas moins de six questions prioritaires de constitutionnalité $(\mathrm{QPC})^{4}$ qui ont abouti à la censure d'importantes dispositions des lois préexistantes en matière d'organisation des soins sans consentement ${ }^{5}$, et qui ont amené le Parlement à agir dans des contraintes temporelles fixées par ce même Conseil constitutionnel ${ }^{6}$.

1. Montesquieu, De l'esprit des lois [1758], Paris, Garnier - Flammarion, 1979, p. 67: «Elles doivent être relatives au physique du pays; au climat glacé, brûlant ou tempéré; à la qualité du terrain, à sa situation, à sa grandeur; au genre de vie des peuples, laboureurs, chasseurs ou pasteurs; elles doivent se rapporter au degré de liberté que la constitution peut souffrir, à la religion des habitants, à leurs inclinations, à leurs richesses, à leur nombre, à leur commerce; enfin elles ont des rapports entre elles; elles en ont avec l'origine, avec l'objet du législateur, avec l'ordre des choses sur lesquelles elles sont établies. C'est dans toutes ces vues qu'il faut les considérer ».

2. Loi no $2011-803$ du 5 juillet 2011 relative aux droits et à la protection des personnes faisant l'objet de soins psychiatriques et aux modalités de leur prise en charge.

3. Loi no 2013-869 du 27 septembre 2013 modifiant certaines dispositions issues de la loi no $2011-803$ du 5 juillet 2011 relative aux droits et à la protection des personnes faisant l'objet de soins psychiatriques et aux modalités de leur prise en charge.

4. CC, déc. $\mathrm{n}^{\circ}$ 2010-71 QPC du 26 novembre 2010, Mlle Danielle S.; déc. $\mathrm{n}^{\circ}$ 2011-135/140 QPC du 9 juin 2011, M. Abdellatif B. et autre; déc. $\mathrm{n}^{\circ} 2011-$ 174 QPC du 6 octobre 2011, $M^{\text {me }}$ Oriette P.; déc. no 2011-185 QPC du 21 octobre 2011, M. Jean-Louis C.; déc. nº $2011-202$ QPC du 2 décembre 2011, $M^{\text {me }}$ Lucienne Q. ; déc. no 2012-235 QPC du 20 avril 2012, Association Cercle de réflexion et de proposition d'actions sur la psychiatrie. Cette impressionnante liste de QPC a toutefois eu un effet pervers en annihilant le contrôle a priori des lois du 5 juillet 2011 et du 27 septembre 2013 : aucune des autorités de saisine compétentes n'a en effet jugé utile de déférer au Conseil constitutionnel le contrôle de la constitutionnalité de ces textes sur le fondement de l'article 61 de la Constitution, estimant probablement que de futures QPC permettront à l'avenir de purger de leurs éléments d'inconstitutionnalité les deux lois..

5. Les six QPC ont essentiellement porté sur des dispositions de la loi n 90-527 du 27 juin 1990, mais elles ont également concerné la loi du 30 juin 1838 sur les aliénés (QPC 2011-202) et la loi du 5 juillet 2011 (QPC 2012-235).

6. Le deuxième alinéa de l'article 62 de la Constitution du 4 octobre 1958 prévoit en effet qu' « une disposition déclarée inconstitutionnelle sur le fondement de l'article 61-1 est abrogée à compter de la publication de la décision du Conseil constitutionnel ou d'une date ultérieure fixée par cette décision". Le Conseil peut ainsi donner un effet différé à ses décisions QPC, faculté utilisée dès sa première QPC (déc. 2010-1 QPC du 28 mai 2010, Consorts L.). Dans sa décision no 2010-71, le Conseil constitutionnel déclare ainsi contraire à la Constitution l'article L. 337 du Code de la santé publique (CSP) et fixe au $1^{\text {er }}$ août 2011 la prise d'effet de cette déclaration d'inconstitutionnalité afin de permettre au législateur d'y remédier. Dans sa décision n 2012-235 QPC, il déclare contraires à la Constitution le paragraphe II de l'article L. 3211-12 CSP et l'article L. 3213-8 et reporte au $1^{\text {er }}$ octobre 2013 la date de leur abrogation. 
Le rôle du Conseil constitutionnel est donc central dans ce mouvement récent de rénovation du droit des hospitalisations psychiatriques. Mais il ne s'agit pas de la seule source d'influence, car les exigences posées par le Conseil, mais aussi le travail parlementaire, démontrent la volonté de conformer le droit français aux standards européens imposés par la Convention européenne de sauvegarde des droits de l'homme et des libertés fondamentales du 4 novembre 1950 et par la jurisprudence de la Cour européenne des droits de l'homme . Certes, le Conseil constitutionnel n'opère officiellement aucun contrôle de conventionnalité ${ }^{8}$, mais la protection par ce même Conseil des «droits et libertés que la Constitution garantit» grâce à la QPC (art. 61-1 de la Constitution) permet d'intégrer en droit interne nombre d'exigences conventionnelles. La lecture des lois du 5 juillet 2011 et du 27 septembre 2013 permet ainsi de découvrir les influences croisées du Conseil constitutionnel et du Conseil de l'Europe. Elles ont conduit à réformer le droit français - de manière plus ou moins intense - sur trois points: en renforçant la légitimité et la proportionnalité des dispositifs d'hospitalisation sans consentement (I), en exigeant que ces mêmes dispositifs soient soumis à des contrôles effectifs (II), et enfin en vérifiant le respect de la dignité des personnes soumises à de telles hospitalisations sans consentement (III).

\section{Limiter les hospitalisations sans consentement à des cas "légitimes" mis en œuvre par des techniques "proportionnées "}

Dès la première QPC relative à l'hospitalisation sans consentement (déc. no 2010-71 QPC du 26 novembre 2010, $M^{\text {lle }}$ Danielle S.), le Conseil pose les termes de son analyse en la matière: si l'hospitalisation sans consentement peut être légitimement prévue par le législateur, elle ne doit pas pour autant présenter une rigueur telle qu'elle entraverait la liberté individuelle de manière excessive. Dans cette décision (même s'il n'effectue en l'espèce qu'un contrôle restreint...), le Conseil vérifie ainsi que la loi Évin du 27 juin 1990, qui prévoit des possibilités d'hospitalisation sans consentement, met en œuvre des techniques assurant une conciliation «qui n'est pas manifestement disproportionnée» entre d'une part la protection de la santé et la sauvegarde de l'ordre public et, d'autre part, la liberté personnelle, protégée par l'article 2 de la Déclaration des droits de l'homme et du citoyen ${ }^{9}$. Cette analyse classique du juge constitutionnel qui doit arbitrer entre différents intérêts de rang constitutionnel ${ }^{10}$ fait clairement écho à la jurisprudence de la Cour européenne des droits de l'homme qui exige, depuis l'arrêt Winterwerp de 1979, que pour toute privation de liberté en matière psychiatrique l' «aliénation » soit établie de manière "probante $»^{11}$. Plus précisément, comme le dit aujourd'hui la Cour dans de nombreuses décisions, trois conditions doivent être réunies: l'aliénation doit avoir été établie de manière probante; le trouble doit revêtir un caractère ou une ampleur légitimant l'internement; enfin, l'internement ne peut se prolonger valablement sans la persistance de pareil trouble $^{12}$. Le respect de ces exigences permet de s'assurer de l'absence d'arbitraire dans les internements psychiatriques. Comme ont eu récemment l'occasion de le rappeler les juges de Strasbourg,

La privation de liberté est une mesure si grave qu'elle ne se justifie que lorsque d'autres mesures, moins sévères, ont été considérées et jugées insuffisantes pour sauvegarder l'intérêt personnel ou public exigeant la détention ${ }^{13}$.

Ce contrôle de la nécessité et de la proportionnalité a permis de purger la législation française de plusieurs types de dispositifs douteux, porteurs de discrimination pour les personnes auxquelles ils avaient vocation à s'appliquer ${ }^{14}$.

7. Dans son Rapport fait au nom de la commission des affaires sociales sur la proposition de loi relative aux soins sans consentement en psychiatrie, $\mathrm{n}^{\circ}{ }_{1284}$, enregistré à la présidence de l'Assemblée nationale le 17 juillet 2013 , le député $\mathrm{D}$. Robiliard relève effectivement que «dès sa version initiale, le projet de loi prévoyait des dispositions visant à prendre en compte les recommandations [sic] émises sur la base de la Convention européenne de sauvegarde des droits de l'homme et des libertés fondamentales».

8. Voir CC, déc. 74-54 DC du 15 janvier 1975, Loi relative à l'interruption volontaire de la grossesse.

9. Voir également la déc. 2011-135/140 QPC du 9 juin 2011, M. Abdellatif B. et autre, cons. 11: «l'hospitalisation d'office est réservée aux cas dans lesquels elle est adaptée, nécessaire et proportionnée à l'état du malade ainsi qu'à la sûreté des personnes ou la préservation de l'ordre public».

10. Voir notamment la déc. $n^{\circ}$ 2008-562 du 21 février 2008, Loi relative à la rétention de sûreté et à la déclaration d'irresponsabilité pénale pour cause de trouble mental, dans laquelle le Conseil rappelle que la loi doit respecter «le principe, résultant des articles 9 de la Déclaration de 1789 et 66 de la Constitution, selon lequel la liberté individuelle ne saurait être entravée par une rigueur qui ne soit nécessaire; qu'il incombe en effet au législateur d'assurer la conciliation entre, d'une part, la prévention des atteintes à l'ordre public nécessaire à la sauvegarde de droits et principes de valeur constitutionnelle et, d'autre part, l'exercice des libertés constitutionnellement garanties; [...] que les atteintes portées à l'exercice de ces libertés doivent être adaptées, nécessaires et proportionnées à l’objectif de prévention poursuivi» (cons. 13).

11. Cour EDH, Winterwerp c. Pays-Bas, 24 octobre 1979, n 6301/73, \$39.

12. Pour des applications récentes, voir Cour EDH, H. L. c. Roumanie, 5 octobre 2004, $\mathrm{n}^{\circ}$ 45508/99; C. B. c. Roumanie, 20 avril $2010, \mathrm{n}^{\circ} 21207 / 03$; Cocaign c. France, 3 novembre 2011, nº 32010/07; Donder et de Clippel c. Belgique, 6 décembre 2011, no 8595/o6; Stanev c. Bulgarie, 17 janvier 2012, n 36760/o6; Glien c. Allemagne, 28 novembre 2013, nº 7345/12. Par ailleurs, la «détention» d'une personne comme malade mental ne sera « régulière » au regard de l'article $5, \$ 1$, e) de la Convention que si elle se déroule dans un hôpital, une clinique ou un autre établissement approprié: voir Cour EDH, Ashingdane c. Royaume-Uni, 28 mai 1985, série A, nº 93 et Pankiewicz c. Pologne, 12 février $2008, \mathrm{n}^{\circ} 34151 / 04$.

13. Cour EDH, R. L. et M.-J. D. c. France, 29 avril 2004, $\mathrm{n}^{\circ} 44568 / 98, \S 116$.

14. L'absence de discrimination des personnes soumises à des soins psychiatriques constituant une autre exigence posée par le Conseil de l'Europe. La Recommandation Rec (2004) 10 du Comité des ministres aux États membres relative à la protection des droits de l'homme et de la dignité des personnes atteintes de troubles mentaux prévoit en effet dans son article 3 (non-discrimination) que «Toute forme de discrimination en raison d'un trouble mental devrait être interdite» et que «Les États membres devraient prendre des mesures appropriées afin d'éliminer la discrimination en raison d'un trouble mental». 
En premier lieu, dans sa décision du 6 octobre 2011 (2011-174, $M^{m e}$ Oriette P.), le Conseil déclare inconstitutionnelles les dispositions permettant au maire d'hospitaliser d'office une personne en cas de péril imminent sur le seul fondement de la «notoriété publique» (art. L. 3213-2 CSP, dans sa rédaction antérieure à la loi du 5 juillet 2011). Pour les juges de la rue de Montpensier, cette mention législative apparaît trop subjective, floue et indéterminée, laissant, faute de pouvoir reposer sur un fondement médical, la part belle à la rumeur ou à la simple réputation. Une telle possibilité ouverte par la loi ne permet pas d'être certain qu'elle soit réservée exclusivement « aux cas dans lesquels elle est adaptée, nécessaire et proportionnée à l'état du malade ainsi qu'à la sûreté des personnes ou la préservation de l'ordre public». Bien qu'implicite, l'influence de la Cour de Strasbourg apparait ici évidente: dans son arrêt R. L. et M.-J. D. c. France de 2004, celle-ci avait en effet rappelé à la France sa jurisprudence constante selon laquelle un individu ne peut passer pour "aliéné» si son aliénation n'a pas été établie de manière probante ${ }^{15}$.

En deuxième lieu, la décision 2011-135/140 QPC du 9 juin 2011, M. Abdellatif $B$. et autre aboutit à déclarer inconstitutionnel le dispositif législatif (alors art. L. 3213-1 CSP) de l'hospitalisation d'office ne prévoyant aucun réexamen de la situation d'une personne ayant fait l'objet d'un certificat médical établi dans les vingt-quatre heures suivant son admission et ne confirmant pas la nécessité de soins en hospitalisation. Selon le Conseil, cette lacune ne permet en effet pas de s'assurer que ce type d'internement est réservé aux cas dans lesquels il est «adapté, nécessaire et proportionné à l'état du malade ainsi qu'à la sûreté des personnes ou la préservation de l'ordre public $»^{16}$.

Enfin, plusieurs décisions ont posé des jalons afin d'éviter la mise en place de techniques excessivement dérogatoires pour les délinquants souffrant de pathologies mentales. Dans sa décision 2011-185 QPC du 21 octobre 2011, M. Jean-Louis C., le Conseil a été amené à censurer l'interprétation par la Cour de cassation de l'article L. 3213-8 CSP (dans sa rédaction antérieure à la loi du 5 juillet 2011) qui régissait la levée de l'hospitalisation d'office des personnes pénalement irresponsables. Dans cette QPC d'octobre 2011, tout en reconnaissant que «la situation particulière» des personnes ayant commis des infractions pénales alors qu'elles étaient atteintes de troubles mentaux justifie des "garanties particulières", le Conseil censure l'interprétation de cette disposition ${ }^{17}$ qui aboutit à subordonner à la décision conforme de deux médecins le pouvoir de l'autorité judiciaire d'apprécier s'il y a lieu d'ordonner la remise en liberté de la personne hospitalisée d'office en violation des articles 64 et 66 de la Constitution ${ }^{18}$. Cette volonté de ne pas soumettre ces personnes ayant commis des infractions en état de trouble mental à des exigences disproportionnées et donc liberticides se retrouve dans la décision 2012-235 QPC du 20 avril 2012, Association Cercle de réflexion et de proposition d'actions sur la psychiatrie (qui porte sur la loi du 5 juillet 2011), dans laquelle le Conseil a déclaré inconstitutionnelle la disposition prévoyant que pour ce type de malade les autorités judiciaires «avisent» immédiatement le préfet qui peut, après avoir ordonné la production d'un certificat médical sur l'état du malade, prononcer une mesure d'admission en soins psychiatriques. Cette technique avait été prévue par le législateur quelles que soient la gravité et la nature de l'infraction commise en état de trouble mental et établissait des règles plus rigoureuses que celles applicables aux personnes soumises à l'obligation de soins psychiatriques. Cette même décision de 2012 a permis au Conseil de censurer l'article L. 3222-3 CSP instaurant des conditions plus strictes concernant la levée d'une mesure d'hospitalisation d'irresponsables pénaux et de personnes ayant séjourné en unité pour malades difficiles (UMD) au motif que, si leur situation justifie des mesures particulières, le législateur doit adopter «les garanties légales contre le risque d'arbitraire encadrant la mise en ouvre de ce régime particulier ». Pour le Conseil, ces dispositions méconnaissent la Constitution en ne prévoyant pas l'information préalable de la personne intéressée, et font donc:

[...] découler d'une hospitalisation en unité pour malades difficiles, laquelle est imposée sans garanties légales suffisantes, des règles plus rigoureuses que celles applicables aux autres personnes admises en hospitalisation complète, notamment en ce qui concerne la levée de ces soins ${ }^{19}$.

On voit ici la logique qui sous-tend le raisonnement du Conseil: l'usage des UMD est potentiellement arbitraire ${ }^{20}$

15. Cour EDH, R. L. et M.-J. D. c. France, $\$ 114$.

16. CC, déc. 2011-135/140 QPC du 9 juin 2011, cons. 10.

17. Comme l'a indiqué S. Slama, cette disposition avait été critiquée lors des débats à l'Assemblée nationale, notamment par M. Dreyfus-Schmidt qui avait évoqué son caractère abusif au moment de son adoption par la loi Évin du 27 juin 1990 ( «Inconstitutionnalité du régime de levée de l'hospitalisation d'office des personnes pénalement irresponsables antérieur à la loi du 5 juillet 2011 », lettre Actualités Droits-Libertés du CREDOF, 27 octobre 2011, en ligne: https://groupes.renater.fr/sympa/arc/droits-libertes/2011-10/msgooo13/Lettre_ADL_du_CREDOF_-_27_octobre_2011.pdf).

18. La loi du 5 juillet 2011 a substitué aux anciennes dispositions de l'article L. 3213-8 CSP de nouvelles dispositions prévoyant que le préfet ne peut décider de mettre fin à une mesure de soins psychiatriques qu'après avis du collège mentionné à l'article L. 3211-9 CSP ainsi qu'après deux avis concordants sur l'état mental du patient émis par deux psychiatres.

19. CC, déc. $\mathrm{n}^{\circ}$ 2012-235 QPC du 20 avril 2012, cons. 26. La loi du 5 juillet 2011 prévoyait pour ces malades un régime spécifique de mainlevée des mesures de soins nécessitant que le juge des libertés et de la détention (JLD) recueille, avant de statuer, l'avis d'un collège d'experts, et ne puisse décider la mainlevée qu'après deux expertises par deux experts différents. La loi du 27 septembre 2013 réserve ces modalités spécifiques aux personnes ayant été considérées pénalement irresponsables à raison de faits punis d'au moins cinq ans de prison en cas d'atteinte aux personnes ou d'au moins dix ans de prison en cas d'atteinte aux biens, soit des peines d'une particulière gravité.

20. Sur certaines modalités de fonctionnement des UMD, voir: Contrôleur général des lieux de privation de liberté, «Avis du 17 janvier 2013 relatif aux séjours injustifiés en unités pour malades difficiles», JORF, 5 février 2013 , texte no 85 . Voir également le commentaire de L. Friouret, «La protection des droits fondamentaux des personnes faisant l'objet d'une hospitalisation sous contrainte en Unité pour Malades Difficiles», lettre Actualités Droits-Libertés du CREDOF, 24 février 2013, en ligne: http://revdh.files.wordpress.com/2013/o2/lettre-adl-du-credof-24-fevrier-2013.pdf. 
ce qui est inacceptable s'agissant d'une mesure grave de nature à restreindre l'exercice de la liberté individuelle ${ }^{21} \ldots$

La rénovation de la législation pertinente a sans nul doute abouti à rationaliser les différentes procédures d'hospitalisation sans consentement. Elle a également permis, grâce à la même double influence explicite et implicite du couple juge constitutionnel/ juge conventionnel, de renforcer les regards extérieurs sur ces procédures, en mettant le juge judiciaire au cœur du dispositif de contrôle.

\section{Des hospitalisations sans consentement effectivement contrôlées: la place essentielle du juge}

Les dispositifs d'hospitalisation sans consentement constituent juridiquement des hypothèses de privation de liberté: dans son arrêt Storck c. Allemagne du 16 juin 2005, la Cour européenne des droits de l'homme nous a rappelé qu'une personne peut passer pour avoir été privée de sa liberté à partir du moment où elle n'a pas valablement consenti à son internement ${ }^{22}$. Ces situations présentent toujours des risques importants d'atteintes aux libertés individuelles des personnes qui y sont soumises et la Cour de Strasbourg s'est toujours attachée à protéger l'individu contre les risques d'arbitraire ${ }^{23}$. En effet,

[...] compte tenu de sa spécificité et de ses effets potentiels sur le bien-être psychologique et physique de la personne qui en fait l'objet, l'internement en hôpital psychiatrique doit être assorti de garanties procédurales et matérielles particulières adaptées à ce type de privation de liberté ${ }^{24}$.

L'État est donc tenu de prendre des mesures offrant une protection effective à ces personnes vulnérables que sont les personnes privées de liberté ${ }^{25}$. Au cœur de cette protection offerte aux personnes privées de leur liberté se situe l'obligatoire contrôle d'un juge indépendant. En vertu de l'article 5 de la Convention européenne des droits de l'homme, ce magistrat doit intervenir lors de la phase initiale de la privation de liberté ( $\operatorname{art} .5, \S_{3}$ ), mais également à un stade ultérieur pour pouvoir statuer sur la légalité de la privation de liberté (art. $5, \$ 4$ ). Dans le cas spécifique des aliénés, la Cour de Strasbourg a fermement rappelé qu'outre le contrôle de la décision privative de liberté il doit toujours y avoir place pour un contrôle ultérieur, à exercer à des intervalles raisonnables, car les motifs qui justifiaient à l'origine la détention peuvent cesser d'exister ${ }^{26}$. Cette exigence d'un juge garant des personnes privées de leur liberté se retrouve dans la jurisprudence du Conseil constitutionnel. Selon lui, en effet, «la liberté individuelle ne peut être tenue pour sauvegardée que si le juge intervient dans le plus court délai possible ${ }^{27}$, puisqu'en vertu de l'article 66 de la Constitution «l'autorité judiciaire est gardienne de la liberté individuelle ${ }^{28}$.

Les différentes QPC adoptées par le Conseil constitutionnel depuis 2010 ont été l'occasion pour lui de transposer aux situations d'hospitalisation sans consentement ses exigences de principe ${ }^{29}$. L'apport de la QPC 2010-71 QPC, $M^{\text {lle }}$ Danielle $S$. du 26 novembre 2010 est à cet égard décisif: mettant en avant le respect de l'article 66 de la Constitution qui fait de l'autorité judiciaire la garante de la liberté individuelle, le Conseil rappelle que cette disposition exige que toute privation de liberté soit placée sous le contrôle de l'autorité judiciaire. En conséquence, même si la Constitution n'impose pas que cette dernière soit saisie préalablement à toute mesure de privation de liberté, il est impossible de maintenir cette hospitalisation au-delà d'un certain délai (en l'occurrence quinze jours) sans intervention d'une juridiction de l'ordre judiciaire (ce que ne prévoyaient pas alors les dispositions de l'article L. 337 CSP). Par ailleurs, le Conseil rappelle que la personne hospitalisée (ou toute personne intéressée) possède un véritable droit au

21. Les UMD étaient prévues par l'article L. 3222-3 CSP qui disposait que «Les personnes faisant l'objet de soins psychiatriques sous la forme d'une hospitalisation complète en application des chapitres III ou IV du titre I ${ }^{\text {er }}$ du présent livre ou de l'article 706-135 du Code de procédure pénale (CPP) peuvent être prises en charge dans une unité pour malades difficiles lorsqu'elles présentent pour autrui un danger tel que les soins, la surveillance et les mesures de sûreté nécessaires ne peuvent être mis en œuvre que dans une unité spécifique». La grande faiblesse du droit des UMD venait du fait qu'il n'existait aucune définition législative et donc prévisible du « $\mathrm{D}$ » et qu'elles n'avaient vocation qu'à accueillir des patients dont le soin était imposé par le préfet. La loi du 5 juillet 2011 avait prévu un dispositif particulier concernant ce type de malades présentant des risques potentiels de dangerosité. Le juge, pour se prononcer, devait en effet disposer de l'avis motivé de deux psychiatres, dont un participant à la prise en charge du patient, ainsi que d'un membre de l'équipe pluridisciplinaire participant à cette prise en charge. En outre, si le juge envisageait d'ordonner une mainlevée de la mesure d'hospitalisation complète, il devait préalablement avoir recueilli les conclusions de deux experts. La loi du 27 septembre 2013 supprime le caractère spécifique des UMD en les replaçant dans le droit commun des services hospitaliers psychiatriques, ce qui a pour effet de donner à nouveau aux patients qui y sont placés les mêmes droits et garanties que dans un service ordinaire.

22. Cour EDH, Storck c. Allemagne, 16 juin 2005, n 61603/oo, $\$ 76$. Voir également Cour EDH, H. L. c. Royaume-Uni, 5 octobre 2004, $\mathrm{n}^{\circ}$ 45508/99, $\$ 89-94$.

23. Cour EDH, Herczegfalvy c. Autriche, 24 septembre 1992, série A, no 244, $\$ 63$.

24. Cour EDH, Ümit Bilgic c. Turquie, 3 septembre 2013, nº 22398/05, \$91.

25. Cour EDH, Storck c. Allemagne, $\$ 102$.

26. Cour EDH, Luberti c. Italie, 23 février 1984, série A, $\mathrm{n}^{\circ} 75, \$ 31$.

27. CC, déc. $n^{\circ}$ 79-109 DC du 9 janvier 1980, Loi relative à la prévention de l'immigration clandestine et portant modification de l'ordonnance $n^{\circ} 45-2658$ du 2 novembre 1945 relative aux conditions d'entrée et de séjour en France des étrangers et portant création de l'office national d'immigration, cons. 4.

28. CC, déc. $\mathrm{n}^{\circ}$ 92-307 DC du 25 février 1992, Loi portant modification de l'ordonnance $n^{\circ}$ 45-2658 du 2 novembre 1945 modifiée relative aux conditions d'entrée et de séjour des étrangers en France, cons. 12. Voir également la déc. nº 2008-562 DC du 21 février 2008, Loi relative à la rétention de sûreté et à la déclaration d'irresponsabilité pénale pour cause de trouble mental.

29. Garanties qui avaient été anticipées par un décret du 20 mai 2010 qui avait enserré l'intervention du JLD dans des délais brefs : l'article R. $3211-9$ CSP impartit au juge un délai de douze jours pour statuer, le délai étant porté à vingt-cinq jours en cas d'expertise, laquelle doit être réalisée dans un délai de quinze jours selon l'article R. 3211-6 du CSP. 
juge, puisqu'elle dispose du droit de saisir à tout moment le tribunal de grande instance pour qu'il soit mis fin à l'hospitalisation sans consentement. Le Conseil a même ici formulé une réserve pour que le juge judiciaire soit tenu de statuer sur la demande de sortie immédiate dans les plus brefs délais compte tenu de la nécessité éventuelle de recueillir des éléments d'information complémentaires sur l'état de santé de la personne hospitalisée. Ces exigences ont ensuite été étendues aux hospitalisations d'office prévues par le préfet sur le fondement de l'article L. 3213-1 CSP (hospitalisation d'office des personnes dont les troubles mentaux nécessitent des soins et compromettent la sûreté des personnes ou portent atteinte, de façon grave, à l'ordre public) dans la décision 2011-135/140 QPC, $M$. Abdellatif $B$. et autre du 9 juin 2011, et aux mesures de "placement volontaire» décidées par le directeur d'un établissement psychiatrique à la demande de toute personne autre que celle visée par la mesure (ancien art. L. 336 CSP, antérieur à la loi du 27 juin 1990) avec la décision n 2011-202 QPC du 2 décembre 2011, $M^{\text {me }}$ Lucienne $Q$.

Cet ensemble de décisions a évidemment eu un impact direct sur la loi du 5 juillet 2011 qui a intégré ces exigences de contrôle en mettant en place (avec le nouvel art. L. 321112-1 CSP) un bloc de compétence au profit du juge judiciaire et en prévoyant qu'aucune décision d'admission en soins psychiatriques sous forme d'hospitalisation complète ne pourra se poursuivre au-delà d'un délai de quinze jours sans que le JLD n'ait statué. Celui-ci doit ensuite intervenir tous les six mois suivant la décision de maintien prononcée par le juge, ou avant l'expiration d'un délai de six mois suivant la décision judiciaire prononçant l'admission en soins psychiatriques sans consentement pour les personnes déclarées pénalement irresponsables, puis tous les six mois. La loi a également mis en place une saisine systématique du juge si, au-delà du quinzième jour de l'admission en hospitalisation complète, le préfet n'ordonne pas la levée d'une mesure de soins sans consentement, alors qu'un psychiatre atteste par certificat médical que les conditions justifiant cette hospitalisation ne sont plus remplies (art. L. 2313-5 CSP) ${ }^{30}$. On a pu s'étonner en
2011 du délai de quinze jours pour l'intervention du juge, lorsque l'on sait que pour d'autres situations de privation de liberté le juge constitutionnel impose un contrôle du juge nettement plus rapide: ainsi, en matière de garde à vue ou de rétention judiciaire, c'est normalement au-delà de quarante-huit heures qu'aucune personne ne peut être retenue contre sa volonté sauf par décision judiciaire ${ }^{31}$; de même, dans sa décision du 9 janvier 1980, le Conseil a censuré des dispositions qui reportaient au septième jour l'intervention judiciaire pour les étrangers retenus ${ }^{32}$. Mais le Conseil constitutionnel a déjà eu l'occasion de préciser que pour la mise en œuvre de l'article 66 de la Constitution, «le législateur peut fixer des modalités d'intervention de l'autorité judiciaire différentes selon la nature et la portée des mesures touchant à la liberté individuelle qu'il entend édicter ${ }^{33}$. On peut également penser que le juge constitutionnel a fait preuve de pragmatisme en ayant présent à l'esprit les spécificités de la situation qui nécessite d'abord une expertise médicale et psychiatrique et qui rendrait sans réel effet un contrôle plus rapide de l'autorité judiciaire (le Conseil dans sa QPC $M^{l l e}$ Danielle $S$. - précitée - mettait en avant les «motifs médicaux» et les «finalités thérapeutiques»). Le juge européen adopte d'ailleurs la même démarche, en jugeant que «dans une procédure de contrôle d'un internement psychiatrique, la complexité des questions médicales en jeu est un facteur pouvant entrer en ligne de compte ${ }^{34}$, ce qui l'a également amené à indiquer depuis longtemps qu'une décision d'admission en établissement psychiatrique peut être prise par une autorité administrative $^{35}$. En effet, la Cour a toujours rappelé la grande latitude dont les États contractants disposent dans des cas d'internement psychiatrique au titre de l'urgence ${ }^{36}$. La loi du 27 septembre 2013 (qui entrera en vigueur sur ce point au $1^{\text {er }}$ septembre 2014) a toutefois ramené ce délai à douze jours (avec un délai de première saisine obligatoire désormais fixé à huit jours à compter de l'admission du patient), afin que le JLD exerce son contrôle plus précocement et puisse plus rapidement mettre fin, le cas échéant, à des mesures qui ne seraient pas pleinement justifiées.

30. Ces recours s'ajoutant aux contrôles qui existaient déjà avant la loi du 5 juillet 2011: art. L. 3211-12 modifié CSP (recours contre une mesure de soins sans consentement); art. L. 3211-12 et R. 3211-14 CSP (contrôle du JLD d'office). Sur toutes ces questions, voir Y. Broussolle, «Les principales dispositions de la loi relative aux droits et à la protection des personnes faisant l'objet de soins psychiatriques et aux modalités de leur prise en charge", Les petites affiches, $\mathrm{n}^{\circ} 212,25$ octobre 2011, p. 5-14.

31. La durée initiale de la garde à vue est de vingt-quatre heures, avec possibilité d'une prolongation de vingt-quatre heures supplémentaires lorsque l'infraction motivant la mesure de garde à vue est punie d'au moins un an d'emprisonnement, soit quarante-huit heures maximum. Cette prolongation doit être justifiée par les nécessités de l'enquête, en principe la prolongation est subordonnée à la présentation du gardé à vue au procureur de la République ou à un juge d'instruction suivant le cas, par exception, la décision écrite et motivée sans présentation préalable de la personne. Depuis la loi nº 2004-204 du 9 mars 2004 portant adaptation de la justice aux évolutions de la criminalité (dite loi «Perben II»), la garde à vue peut durer jusqu'à quatre-vingt-seize heures pour un certain nombre d'infractions mentionnées à l'article 706-73 CPP. Enfin, la garde à vue peut également être prolongée et durer six jours pour les besoins de l'enquête relative au terrorisme lorsqu'il y a un risque actuel d'actes de terrorisme.

32. Annabelle Pena rappelle également que le juge italien est saisi dans les quarante-huit heures qui suivent la décision initiale d'hospitalisation sans consentement tandis que le juge espagnol doit l'être à l'issue des vingt-quatre premières heures lorsque l'hospitalisation a été décidée en urgence. Voir A. Pena, «Internement psychiatrique, liberté individuelle et dualisme juridictionnel: la nouvelle donne», Revue française de droit administratif, $\mathrm{n}^{\circ}$ 5, 2011, p. 957.

33. CC, déc. n० 92-307 DC du 25 février 1992, cons. 13.

34. Cour EDH, Musial c. Pologne, 25 mars 1999, n² 24557/94, $\$ 47$.

35. Cour EDH, X. c. Royaume-Uni, 5 novembre 1981, $\mathrm{n}^{\circ} 7215 / 75, \S 55 s q$

36. Cour EDH, Varbanov c. Bulgarie, 5 octobre 2000, $\mathrm{n}^{\circ} 31365 / 96, \S 58 \mathrm{sq}$ 
La chronologie des réformes adoptées en la matière est tout à fait révélatrice de l'influence de la Cour européenne dans cette extension des garanties juridictionnelles: la décision QPC du 26 novembre 2010 est en effet rendue quelques jours à peine après une condamnation de la France par la Cour de Strasbourg dans son arrêt Baudoin c. France du 18 novembre 2010. Elle avait alors jugé que:

[...] l'articulation entre la compétence du juge judiciaire et celle du juge administratif quant aux voies de recours offertes n'a pas permis au requérant d'obtenir une décision d'un tribunal pouvant statuer «sur la légalité de sa détention et ordonner sa libération si la détention est illégale ${ }^{37}$.

La France avait donc violé le droit au recours effectif du requérant ${ }^{38}$. Depuis 2002 , le recours pour excès de pouvoir exercé devant le juge administratif n'est en effet pas considéré comme un recours pertinent en la matière, puisqu'il ne permet pas d'obtenir une remise en liberté ${ }^{39}$. De même, la QPC du 9 juin 2011 intervient quelques jours à peine après une nouvelle condamnation strasbourgeoise dans l'affaire Patoux c. France, pour laquelle la Cour juge qu'en examinant une demande de sortie immédiate quarante-six jours après avoir été saisi, le JLD n'a pas statué à «bref délai » sur la légalité de son internement, comme l'exige l'article $5, \$ 4$ de la Convention (la requérante avait fait l'objet d'une mesure d'hospitalisation d'office provisoire par le maire au centre hospitalier interdépartemental) ${ }^{40}$.

Malgré ces modifications législatives, on peut toutefois se demander si le droit français ne se situe toujours pas en retrait par rapport aux exigences conventionnelles au moins sur un point. Dans sa décision précitée du 20 avril 2012, le Conseil constitutionnel précise en effet que la garantie apportée par la surveillance du JLD ne concerne que les situations de soins prenant la forme d'une hospitalisation complète. En d'autres termes, le contrôle de plein droit de l'autorité judiciaire ne s'applique pas aux soins ambulatoires (simple « restriction» et non «privation» de liberté), même si ceux-ci sont associés à une hospitalisation à temps partiel. Le Conseil refuse de voir ici que le refus de soins peut conduire à une hospitalisation complète... et donc devenir alors une véritable mesure de privation de liberté. Or, dans son arrêt Stanev c. Bulgarie du 17 janvier 2012, la Cour européenne des droits de l'homme a retenu une analyse sensiblement différente: elle assimile en effet une mesure de placement en foyer social pour personnes atteintes de troubles mentaux à une privation de liberté, puisque ce placement, tout en permettant à l'intéressé de sortir de son foyer, emporte des «restrictions importantes à [s]a liberté individuelle». L'absence de contrôle systématique du juge judiciaire sur les situations de soins sans hospitalisation complète pourrait donc s'analyser à la lumière de cette jurisprudence européenne comme un défaut de garantie ne respectant pas les exigences de l'article $5, \S 1$, e) de la Convention européenne des droits de l'homme.

Décisives quant à la rationalisation des procédures d'hospitalisation sans consentement et quant à la nécessité d'instaurer des contrôles effectifs ${ }^{41}$, les différentes influences extérieures ont par contre moins pesé sur le travail du législateur lorsqu'il s'est agi de renforcer la dignité de la personne soumise à ces soins sans consentement.

\section{La question de la dignité de la personne soumise à des soins sans consentement}

L'arrêt Tomasi c. France du 27 août 1992 a permis à la Cour européenne des droits de l'homme de poser un principe nouveau: à l'égard d'une personne privée de sa liberté, tout usage de la force physique qui n'est pas rendu strictement nécessaire par le propre comportement de ladite personne porte atteinte à la dignité humaine ${ }^{42}$ et cette exigence - qui est surtout connue pour avoir produit une considérable jurisprudence en matière pénitentiaire ${ }^{43}$ - a également entraîné plusieurs applications concernant le traitement des personnes souffrant de pathologies mentales ${ }^{44}$. Cette exigence s'avère d'autant plus importante qu'il s'agit de

37. Cour EDH, Baudoin c. France, 18 novembre 2010, $n^{\circ}$ 35935/03, $\$ 108$.

38. Ibid., $\$ 10$. Le Conseil d’État a, dans sa décision $n^{\circ} 335753$ du $1^{\text {er }}$ avril 2010, également considéré que le juge des référés libertés, habilité par l'article L. 521-2 du Code de justice administrative (CJA) à ordonner dans les quarante-huit heures toutes mesures nécessaires à la sauvegarde d'une liberté fondamentale à laquelle une personne morale de droit public aurait porté dans l'exercice d'un de ses pouvoirs une atteinte grave et manifestement illégale, peut se prononcer sur la régularité d'une mesure d'hospitalisation sans consentement, par nature privative de liberté d'aller et de venir, même si cela le conduit à examiner le bien-fondé de la mesure et ainsi à exercer un office proche de celui du juge judiciaire.

39. Cour EDH, Delbec c. France, 18 juin 2002, nº 43125/98.

40. Cour EDH, Patoux c. France, 14 avril 2011, n 35079/06. La Cour a également sanctionné la France dans d'autres arrêts, en n'acceptant notamment pas que le juge judiciaire, saisi d'une demande de sortie immédiate, désigne un expert trois mois plus tard, alors que la mesure d'internement avait été levée une semaine auparavant (D. M. c. France, 27 juin 2002, n 41376/98). Voir également Delbec c. France, précité; Mathieu c. France, 27 octobre $2005, \mathrm{n}^{\circ} 59450 / 00$.

41. Au-delà du contrôle essentiel opéré par le juge judiciaire, la loi du 27 septembre 2013 insère dans le CSP un nouvel article L. 3222-4-1 qui autorise les députés, les sénateurs et les membres du Parlement européen élus en France à visiter à tout moment les établissements où sont dispensés des soins psychiatriques sans consentement. Ce nouveau dispositif de contrôle, inspiré de ce qui existe déjà pour les établissements pénitentiaires (voir l'art. 719 CPP) reste toutefois assez flou quant à ses modalités concrètes de mise en œuvre, puisque rien n'est dit sur ce qu'il adviendrait à la suite d'éventuelles constatations formulées par ces parlementaires après une visite dans un établissement.

42. Cour EDH, Tomasi c. France, 27 août 1992, série A, $\mathrm{n}^{\circ} 241$, $\$ 115$. Voir également les arrêts Ribitsch c. Autriche, 4 décembre 1995 , série A, nº 336 , $\$ 38$; Tekin c. Turquie, 9 juin 1998, n 41556/98, $\$ 53$. Ce principe a également été consacré au plan international par l'article 10 du Pacte international relatif aux droits civils et politiques du 16 décembre 1966, selon lequel «Toute personne privée de sa liberté est traitée avec humanité et avec le respect de la dignité inhérente à la personne humaine».

43. Voir notamment le très important arrêt Kudla c. Pologne (GC), 2 octobre 2000, $\mathrm{n}^{\circ}$ 30210/96.

44. Voir, inter alia, Cour EDH, Aerts c. Belgique, 30 juillet 1998, n 61/1997/845/1051; Filip c. Roumanie, 14 décembre $2006, \mathrm{n}^{\circ} 41124 / 02$. 
personnes vulnérables, parfois incapables de se plaindre des effets d'un traitement appliqué sur leur personne ${ }^{45}$.

Dès sa première QPC relative aux soins sans consentement ( $M^{l l e}$ Danielle S., précitée), le Conseil constitutionnel a également été amené à se prononcer sur la question du respect de la dignité des personnes hospitalisées sans leur consentement. Il a alors suivi la même démarche que celle adoptée par la Cour de Strasbourg et a appliqué aux personnes soignées sans leur consentement les exigences qu'il avait déjà formulées concernant les personnes gardées à $v^{4}{ }^{46}$. Suivant sa position adoptée dans la décision $n^{\circ} 2009$ 593 DC du 19 novembre 2009 sur la loi pénitentiaire, le Conseil a rappelé le principe de dignité de la personne humaine. Mais il s'est ensuite contenté d'énumérer les garanties posées alors par l'article L. 326-3 CSP (actuellement article L. 3211-3 CSP) en rappelant que cette disposition confie aux professionnels de santé et à de nombreuses autorités administratives et judiciaires le soin de veiller, dans le cadre de leurs compétences respectives, au respect de la dignité de la personne, et que la loi prévoit également la répression des éventuels manquements à ce principe. Par ailleurs, le Conseil rappelle que cette même disposition protège également une longue liste de droits garantis «en tout état de cause ${ }^{47}$. Ce faisant, il effectue en l'espèce son travail comme un strict juge de la constitutionnalité ${ }^{48}$ : il ne peut être reproché au législateur de méconnaître le principe de dignité, puisqu'il a, bien au contraire, tenté de poser un certain nombre de garanties visant à le protéger. Le Conseil montre ici ses limites en tant que «juge des droits de l'homme», puisqu'il ne peut condamner les agissements et pratiques de soignants ou d'institutions psychiatriques qui, dans leur action quotidienne et concrète, méconnaîtraient la dignité de la personne hospitalisée sans son consentement. La jurisprudence constitutionnelle apparaît alors forcément en retrait par rapport aux solutions de la Cour de Strasbourg qui a notamment rappelé récemment dans l'arrêt Stanev c. Bulgarie du 17 janvier 2012 (précité) que:

[...] le bien-être d'une personne atteinte de troubles mentaux peut constituer un facteur [...] à prendre en compte, en plus des éléments médicaux, lors de l'évaluation de la nécessité de placer cette personne dans une institution ${ }^{49}$.

Il convient toutefois de souligner l'apport de la loi du 27 septembre 2013 qui, en renforçant un certain nombre de garanties procédurales (nouvel art. L 3211-12-2 CSP), contribue à faire des malades de véritables requérants, qui nécessitent des mesures d'adaptation spécifiques: alors que la loi de 2011 posait le principe d'une audience au siège du tribunal de grande instance (TGI) et, exceptionnellement, dans une salle dédiée de l'établissement lorsque cela était possible, la loi de 2013 (qui n'entre en vigueur sur ces points qu'au $1^{\text {er }}$ septembre 2014) fait de cette salle (qui peut être aménagée au sein de l'établissement d'accueil ou mutualisée avec d'autres établissements) le lieu de déroulement normal, l'audience au TGI devenant l'exception ${ }^{50}$. Le caractère potentiellement traumatisant du déplacement au tribunal pour des personnes fragiles n'est donc plus la norme. Par ailleurs, les débats sont par principe publics mais peuvent avoir lieu en chambre du conseil, de droit lorsque la personne faisant l'objet de soins psychiatriques ou son représentant le demande ou si le juge le décide, cette disposition permettant de mieux faire respecter le droit à la vie privée du malade dans des cas particulièrement difficiles. On doit aussi relever que si la loi de 2011 prévoyait la possibilité pour le patient d'être assisté d'un avocat «le cas échéant», la formulation retenue dans la loi de 2013 en fait désormais un principe. Cette dernière garantie apparaît d'autant plus indispensable que le requérant concerné est une personne dont le jugement est souvent altéré et n'est pas le mieux à même de faire respecter ses droits ${ }^{51}$

Si la dignité de la personne hospitalisée sans consentement n'est pas effectivement protégée par le Conseil qui refuse d'examiner les conditions matérielles de l'internement, on ne saurait toutefois ignorer qu'il a tout de même participé à la protection de ce principe par un autre biais. Dans la QPC précitée 2010-71 du 26 novembre 2010 ( $M^{\text {le }}$ Danielle S.), la requérante estimait en effet que c'est le principe même des hospitalisations sans consentement qui est attentatoire à la dignité humaine, en ne reconnaissant pas à ces personnes le droit de refuser un traitement. On sait en effet que le Conseil d'État a jugé en 2002 que le droit pour un patient majeur de donner, lorsqu'il se trouve en état de l'exprimer, son consentement à un traitement médical revêt le caractère d'une liberté fondamentale ${ }^{52}$ et que les restrictions à l'exercice de ce droit doivent donc être limitées et encadrées. Or, la question du respect du consentement est balayée en quelques mots par le Conseil

45. Cour EDH, Keenan c. Royaume-Uni, 3 avril 2001, $\mathrm{n}^{\circ}$ 27229/95, $\$ 111$; Rivière c. France, 11 juillet 2006, $\mathrm{n}^{\circ} 33834 / 03$

46. CC, déc. $\mathrm{n}^{\circ}$ 2010-14/22 QPC du 30 juillet 2010, M. Daniel W. et autres. Ce qui peut d'ailleurs sembler assez étonnant de la part d'un Conseil constitutionnel qui n'a pas hésité à mettre en avant des exigences sanitaires et de soins pour ne pas exiger un contrôle précoce du juge judiciaire, voir supra.

47. Ce qui comprend notamment le droit de prendre conseil auprès d'un avocat ou d'un médecin de son choix, celui de communiquer avec le directeur général de l'agence régionale de santé, le procureur de la République, le préfet et le maire, celui de saisir la commission départementale des hospitalisations psychiatriques, celui d'émettre ou de recevoir des courriers ou encore celui de consulter le règlement intérieur de l'établissement.

48. Ce qu'il ne fait pas toujours, comme le montre notamment la déc. no 2010-14/22 QPC du 30 juillet 2010, au sujet de la garde à vue.

49. Cour EDH, Stanev c. Bulgarie, $\$ 153$.

50. Ce même article L. 3211-12-2 CSP interdit de recourir à la visioconférence à compter du $1^{\text {er }}$ septembre 2014

51. Sur ces points voir M. Primevert, «Le contrôle du juge sur les soins psychiatriques sans consentement ", La semaine juridique, éd. G, n 22 , 27 mai 2013, doctrine $\mathrm{n}^{\circ} 157$.

52. CE, ord., V. Feuillatey et I. Feuillatey, 16 août 2002, n $^{\circ}$ 249552. Sur cette question du consentement, voir également É. Péchillon, «Le pouvoir médical face au refus de consentement: un savant dosage effectué par le juge des référés ", La semaine juridique, édition administrations et collectivités territoriales, $\mathrm{n}^{\circ} 40,8$ octobre 2012 , chron. $\mathrm{n}^{\circ} 2321$. 
dans la décision $M^{\text {lle }}$ Danielle $S$., puisqu'il se contente d'indiquer de manière assez elliptique que «les garanties encadrant l'hospitalisation sans consentement permettent que l'avis de la personne sur son traitement soit pris en considération ${ }^{53}$. Cela signifie que la mise en place par le législateur de dispositifs d'hospitalisation sans consentement (à la fois pour soigner une personne qui nécessite une "surveillance constante» et pour protéger l'ordre public) est en soi légitime pour le Conseil. L'exigence du consentement ne joue donc pas pleinement son rôle à l'égard des personnes atteintes de graves troubles mentaux. Cette décision du 26 novembre 2010 n'a évidemment pas épuisé les débats autour de la question du consentement, qui a également constitué l'un des éléments soumis à la discussion du Conseil dans la décision 2012-235 du 20 avril 2012 précitée. Celle-ci précise que lorsqu'un patient est soumis

[...] à une obligation de soins psychiatriques pouvant comporter, le cas échéant, des séjours en établissement, les dispositions de l'article L. 3211-2-1 n'autorisent pas l'exécution d'une telle obligation sous la contrainte; que ces personnes ne sauraient se voir administrer des soins de manière coercitive ni être conduites ou maintenues de force pour accomplir les séjours en établissement prévus par le programme de soins $[\ldots]^{54}$.

Si le libellé de ce considérant a pu laisser perplexes certains commentateurs ${ }^{55}$, il signifie plus clairement que pour le Conseil la législation française n'autorise pas l'exécution de l'obligation de soins psychiatriques sous la contrainte en dehors des situations d'hospitalisation complète. Avec la loi du 27 septembre 2013, l'article L. 3211-2-1 CSP est rédigé de manière plus précise et il énumère désormais les différentes modalités de prise en charge: hospitalisation complète, soins ambulatoires, soins à domicile dispensés par un établissement psychia- trique, séjours à temps partiel ou de courte durée à temps complet dans ces mêmes établissements. Et conformément à la volonté du Conseil constitutionnel, ce même article précise explicitement que seules les hospitalisations complètes permettent des mesures de contrainte. Mais ni le Conseil ni le législateur n'ont supprimé toutes les ambiguïtés : en effet, s'il est impossible d'imposer des soins à un patient qui n'est pas en hospitalisation complète, il reste toujours possible aux soignants de faire comprendre à ce malade réfractaire que pèse sur lui, s'il persiste dans son refus, un tel régime d'hospitalisation complète, qui permettra alors d'administrer les soins de manière coercitive ${ }^{56} \ldots$

L'évolution législative récente en matière de soins sans consentement nous offre l'image d'un législateur «sous pression», qui a dû intégrer dans un laps de temps contraint des exigences multiples ${ }^{57}$, ce qui n'est pas allé sans difficultés techniques ${ }^{58}$ ni remous au sein des acteurs du processus législatif ${ }^{59}$. Mais les deux lois du 5 juillet 2011 et du 27 septembre 2013 démontrent aussi de manière plus positive l'importance des influences croisées de la Cour européenne des droits de l'homme et du Conseil constitutionnel lorsqu'il s'agit de moderniser des dispositifs législatifs mettant en cause des droits et libertés. Les six QPC, qui constituent la source la plus directe de l'influence législative, ont ainsi permis au Conseil constitutionnel de s'approprier - et même de compléter - la démarche suivie depuis maintenant plus de trente ans par des juges de Strasbourg qui cherchent à protéger les personnes privées de leur liberté (et notamment celles concernées par l'enfermement psychiatrique) contre l'arbitraire. Les solutions dégagées par les juges de Strasbourg depuis l'arrêt Winterwerp de 1979 (précité) ont directement influencé la jurisprudence constitutionnelle française, qui participe également désormais à la protection des «aliénés» (pour reprendre le vocabulaire de la Convention

53. CC, déc. n 2010-71 QPC du 26 novembre 2010, cons. 32.

54. CC, déc. no 2012-235 QPC du 20 avril 2012, cons. 12.

55. Voir notamment É. Péchillon, «Censure partielle de la loi du 5 juillet 2011 relative aux soins sous contrainte: vers une réforme en profondeur de la psychiatrie avant octobre 2013 ? ", La semaine juridique, édition administrations et collectivités territoriales, $\mathrm{n}^{\circ} 26,2$ juillet 2012 , chron. $\mathrm{n}^{\circ} 2230$.

56. Le commentaire de la décision 2012-235 QPC (précitée) publié sur le site Internet du Conseil constitutionnel (http://www.conseil-constitutionnel. fr/conseil-constitutionnel/root/bank/download/2012235QPCccc_235qpc.pdf) a également pointé les limites du mécanisme: «si la personne ne coopère pas avec le service de soins pour suivre le traitement, se rendre ou demeurer dans l'établissement, il est possible que soit proposé un passage en hospitalisation complète (dans laquelle l'administration de soins contraints sera possible, le régime de protection systématique par le juge étant renforcé par l'intervention systématique de ce dernier après un délai de quinze jours)», p. 7.

57. Et qui est même allé au-delà de ces exigences explicitement posées par le Conseil constitutionnel dans la loi du 27 septembre 2013 : «L'Assemblée nationale et le Gouvernement ont fait le choix de compléter les dispositions répondant à la décision d'avril 2012 du Conseil constitutionnel par des dispositions réformant la loi de 2011 et très attendues par les acteurs du secteur "; J. Le Menn, Rapport fait au nom de la commission des affaires sociales sur la proposition de loi, adoptée par l'Assemblée nationale après engagement de la procédure accélérée, visant à modifier certaines dispositions issues de la loi $n^{\circ} 2011-803$ du 5 juillet 2011 relative aux droits et à la protection des personnes faisant l'objet de soins psychiatriques et aux modalités de leur prise en charge, n 835, enregistré à la présidence du Sénat le 11 septembre 2013, session extraordinaire 2012-2013, p. 10.

58. On sait notamment que la décision du 9 juin 2011 précitée a été adoptée (problème de l'examen contradictoire d'une divergence entre le psychiatre et l'autorité publique) alors que le Sénat effectuait la deuxième lecture de la future loi du 5 juillet 2011. La Haute Assemblée a dû adopter un article ajouté par le gouvernement pour répondre à cette dernière QPC (art. 3 bis).

59. Guy Lefrand, rapporteur de la commission des affaires sociales de l'Assemblée nationale, avait ainsi eu l'occasion de dénoncer cette situation obligeant le Parlement à "légiférer sous la pression du juge constitutionnel sans disposer d'aucun délai de réflexion", dénonçant un tel état de fait "préjudiciable au bon fonctionnement de nos institutions»; G. Lefrand, Rapport fait au nom de la commission des affaires sociales sur le projet de loi, modifié par le Sénat en deuxième lecture, relatif aux droits et protection des personnes faisant l'objet de soins psychiatriques et aux modalités de leur prise en charge, $\mathrm{n}^{\circ} 3546$, enregistré à la présidence de l'Assemblée nationale le 21 juin 2011, p. 5. Voir également G. Drago, «L'influence de la QPC sur le Parlement ou la loi sous la dictée du Conseil constitutionnel», Jus Politicum, n 6, 2011, http://www.juspoliticum. com/L-influence-de-la-QPC-sur-le.html?artpage=3-3. 
de 1950). En s'appropriant ces éléments conventionnels, le Conseil constitutionnel permet que les « deux contrôles (celui de la Cour de Strasbourg et celui du Conseil) se renforcent mutuellement dans leur approche en procurant à l'édifice une véritable cohérence d'ensemble ${ }^{60}$. Cette matière reste toutefois complexe et sensible, car elle repose sur un équilibre délicat rappelé par le député Denys Robiliard: protéger tout à la fois la santé, la garantie des libertés et la sécurité ${ }^{61}$. Il n'est à cet égard pas certain que les réformes mises en place par les lois du 5 juillet 2011 et du 27 septembre 2013 aient réussi à instaurer un équilibre pérenne dans ce domaine ${ }^{62}$.

60. K. Blay-Grabarczyk, «Le régime de l'hospitalisation sous contrainte», Revue française de droit administratif, $\mathrm{n}^{\circ} 4,2012, \mathrm{p} .629$.

61. D. Robiliard, Rapport fait au nom de la commission des affaires sociales..., n $\mathrm{n}^{\circ} 1284$.

62. Éric Péchillon évoque ainsi (sans que cette liste soit limitative) un certain nombre de points susceptibles de poser des problèmes de constitutionnalité : pouvoirs du procureur pouvant faire appel des décisions de mainlevée prononcées par le JLD sachant que cet appel est suspensif en cas de risque d'atteinte grave à l'intégrité du malade ou d'autrui (art. L. 3211-12-4, $3^{\circ}$ et $4^{\circ} \mathrm{CSP}$ ); pouvoirs de ce même juge disposant de la possibilité de retarder de vingt-quatre heures les effets de sa décision en invoquant l'existence de circonstances exceptionnelles pour permettre à l'autorité administrative de s'organiser (art. L. 3211-12-1, IV CSP). La technique du "casier psychiatrique», consistant à signaler au préfet les patients ayant séjourné un an en UMD ou ceux suivis après une déclaration d'irresponsabilité pénale (art. L. 3213-1, I, al. 2 CSP), l'absence d'alternative à l'hospitalisation complète des détenus (art. L. 3214-1 CSP) est jugée «non sérieuse», ou encore les mécanismes des articles L. 3213-4, al. 3 et L. 3216-1, al. 2 CSP. Voir É. Péchillon, «Nouvelle saisine du Conseil constitutionnel à propos de la réforme du soin sous contrainte: les limites de la QPC en matière de protection des libertés", La semaine juridique, édition administrations et collectivités territoriales, $\mathrm{n}^{\circ} 7,20$ février 2012 , act. $\mathrm{n}^{\circ} 106$. 